\title{
Hospital-dia (HD) para transtornos alimentares: revisão da literatura e primeiras impressões do HD do ambulatório de bulimia e transtornos alimentares do IPqHCFMUSP (AMBULIM)
}

Fábio Tapia Salzano ${ }^{1}$

Táki Athanássios Cordás²

Recebido: 20/5/2003 Aceito: 5/6/2003

\section{RESUMO}

Os autores revisaram a literatura a respeito de tratamento em hospital-dia (HD) para transtornos psiquiátricos em geral e mais especificamente para transtornos alimentares, apontando aspectos históricos, indicações e contraindicações para essa forma de tratamento, eficácia clínica e vantagens financeiras. Também foram descritos programas de HD em atividade no mundo, incluindo o HD do AMBULIM-IPqHCFMUSP.

Unitermos: Transtorno psiquiátrico; Transtorno alimentar; Tratamento; Internação parcial; Hospital-dia.

\section{ABSTRACT}

Day hospital care for eating disorders: review of the literature and preliminary findings at AMBULIM, Institute of psychiatry, University of São Paulo

The authors revised the literature about treatment in day-hospital (DH) of psychiatric disorders in general and more specifically about eating disorders, pointing out historical aspects, indications and contra-indications of DH treatment, clinical efficacy and financial advantages. They also described DH programs around the world, including the DH of AMBULIM-IPqHCFMUSP (São Paulo, SP, Brasil).

Keywords: Psychiatric disorder; Eating disorder; Treatment; Partial hospitalization; Day hospital.

\section{Introdução}

$\mathrm{O}$ tratamento dos transtornos alimentares (anorexia nervosa, bulimia nervosa e transtorno do comer compulsivo) tem sido extensamente relatado na literatura.

Não obstante o volume de informações disponíveis, em particular nos últimos 30 anos, residem muitas dúvidas sobre a eficácia dos diferentes procedimentos terapêuticos propostos.

A introdução do tratamento em hospital-dia (HD) para anorexia nervosa e bulimia nervosa é relativamente nova, constituindo-se em uma busca de aumentar a possibilidade de sucesso no tratamento desses quadros considerados, em geral, de mau prognóstico.

Esse artigo faz inicialmente uma revisão da literatura a respeito do tratamento em hospital-dia para transtornos psiquiátricos em geral e, posteriormente, de maneira mais específica, para transtornos alimentares.

A revisão bibliográfica foi feita com base em pesquisa nos seguintes bancos de dados eletrônicos:

MEDLINE - Antigo Index Medicus informatizado, gerenciado pela National Library of Medicine, em Washington, Estados Unidos.

\footnotetext{
Trabalho elaborado no Ambulatório de Bulimia e Transtornos Alimentares do IPqHCFMUSP - AMBULIM.

Psiquiatra Assistente e Coordenador do HD-AMBULIM-IPqHCFMUSP e Pós-graduando do Departamento de Psiquiatria da FMUSP.

Coordenador Geral do AMBULIM-IPqHCFMUSP, Professor Colaborador do Departamento de Psiquiatria da FMUSP.

Endereço para correspondência:

Fábio Tapia Salzano

Ambulatório de Bulimia e Transtornos Alimentares do IPqHCFMUSP (AMBULIM)

Rua Dr. Ovídio Pires de Campos sem nº, Enfermaria feminina, 4º andar - São Paulo, SP - CEP 05403-010

Fonefax: 3069-6975

E-mail: ftsalzano@uol.com.br
} 
EMBASE - Base informatizada da Excerpta Medica.

LILACS - Base de literatura latino-americana em Ciências da Saúde, gerenciada pela Biblioteca Regional de Medicina, em São Paulo (BIREME).

PsycLIT - Base de dados da Associação Americana de Psicologia.

A pesquisa adicional foi feita manualmente no International Journal of Eating Disorders.

As palavras-chave pesquisadas foram: psychiatric disorders, eating disorders, treatment, partial hospitalization e day hospital.

Incluímos, na pesquisa, estudos controlados, comparando tratamento em HD com tratamento em enfermaria de 24 horas ou em ambulatório, bem como programas de atendimento em HD, não controlados, uma vez que é pequeno o número de trabalhos existentes sobre o assunto.

Descrevemos ainda, brevemente, o modelo de atendimento em HD do Ambulatório de Bulimia e Transtornos Alimentares do Instituto de Psiquiatria do Hospital das Clínicas da Faculdade de Medicina da Universidade de São Paulo (AMBULIM-IPqHCFMUSP), em funcionamento desde o final de 1999.

\section{Hospital-dia (HD) para transtornos psiquiátricos}

O primeiro trabalho publicado sobre $\mathrm{o}$ atendimento de pacientes psiquiátricos em HD foi o de Dzhagarova, em 1937, no hospital psiquiátrico de Moscou (Craft, 1959). O autor referiu ter tratado 1.225 pacientes nos primeiros quatro anos de funcionamento do Serviço, sendo $47 \%$ com diagnóstico de esquizofrenia, $38 \%$ de outras psicoses, $6 \%$ com psicose maníaca depressiva e os $9 \%$ restantes de quadros diversos (transtornos de personalidade, neuroses de ansiedade e reações depressivas neuróticas). Não são oferecidos maiores detalhes sobre o programa executado ou os resultados obtidos.

Em 1946, iniciou-se o atendimento em HD em Montreal, no Canadá (Cameron, 1947), de pacientes com depressão, hipomania e esquizofrenia. $\mathrm{O}$ autor sugere que, na presença de ideação suicida ou auto e heteroagressividade, o tratamento em HD está contraindicado sendo a internação em enfermaria a melhor solução. Esse parece ser o primeiro esboço de estabelecer critérios para o uso do HD (Cameron, 1947).

Para Craft (1959), HD deve ser definido como um local onde o tratamento é feito de forma abrangente, com supervisão médica e retorno do paciente para sua casa, à noite. Revisando a literatura sobre os HDs em funcionamento na Inglaterra no ano de 1956, Craft observou que pacientes com vários tipos de alterações psiquiátricas foram atendidos, sem diferenças clínicas nos resultados do tratamento em HD em comparação com as enfermarias, sem, no entanto, apresentar os resultados. Sugere que os principais limitantes para a inclusão do paciente eram a distância da moradia do paciente em relação ao HD e situações familiares tempestuosas (Craft, 1959).

A American Association for Partial Hospitalization (AAPH) define hospitalização parcial como um programa de tratamento semelhante ao ambulatorial que utiliza formas de tratamentos psiquiátricos, psicoterápicos, sociais e pré-vocacionais para pacientes com importantes distúrbios mentais que requerem tratamento multidisciplinar, abrangente e intensivo, não disponível nos modelos ambulatoriais tradicionais (Casarino et al., 1982).

$\mathrm{O}$ atendimento em HD é descrito para diferentes condições psiquiátricas, como esquizofrenia (Gudeman et al., 1983), abuso de substâncias (Fox e Lowe, 1968), deficiência mental (Neisworth e Feeg, 1979), pacientes geriátricos (Arie, 1975) e transtornos alimentares (Piran e Kaplan, 1990), sem no entanto uma avaliação diferencial em cada transtorno.

Nos objetivos do tratamento constam melhorar a transição do regime de internação para o lar, acelerando o retorno à comunidade, mesmo ainda sem remissão do quadro, bem como prevenir recaídas para aqueles que já estavam sendo atendidos ambulatorialmente e tiveram agravamento no quadro, evitando a hospitalização completa.

Esse tipo de atendimento visa encorajar a independência e a responsabilidade do paciente, bem como reduz a permanência em enfermarias de 24 horas, sem prejuízo do tratamento (Gudeman et al., 1983). Recomenda-se, ainda, para o sucesso do regime de HD a existência de uma unidade emergencial de referência e um local de pernoite para os pacientes que não têm família ou moram em outras cidades, o que diminui a possibilidade de se internar um paciente por falta de um local para ele dormir (Gudeman et al., 1983).

Horvitz-Lennon et al. (2001) em uma revisão sobre trabalhos publicados, comparando-se tratamento em HD com internação tradicional, corroboram que a melhora nos dois tratamentos é semelhante nos parâmetros psicopatológicos estudados, no funcionamento social e no familiar. O grau de satisfação com o tratamento avaliado pelo paciente e seus familiares tende a ser maior no grupo que fez HD (Horvitz-Lennon et al., 2001).

Sledge et al. (1996-a) compararam 93 pacientes que participaram de HD com 104 pacientes que ficaram internados em enfermaria psiquiátrica, com alocação randomizada. Os pacientes do HD preenchiam critérios 
para transtornos afetivos em $52 \%$ dos casos $(\mathrm{n}=49)$, quadros psicóticos em $39 \%(\mathrm{n}=36)$ e transtornos ansiosos em $2 \%(n=2)$. Com relação aos pacientes internados, $52 \%(\mathrm{n}=54)$ preenchiam critérios para quadros psicóticos, $44 \%(\mathrm{n}=46)$ para transtornos afetivos e $2 \%$ $(\mathrm{n}=2)$ para transtornos ansiosos. O tempo médio de permanência foi de 26 dias nos dois grupos (Sledge et al., 1996-a).

A análise de aspectos de funcionamento clínicos e sociais, além da qualidade de vida e satisfação com o tratamento, não evidenciou diferenças significativas nos dois grupos ao final do tratamento (Sledge et al., 1996-a).

O custo total do tratamento dos pacientes psicóticos que iam ao HD foi de 25.731 dólares e o dos que estavam internados, 29.342 dólares (Sledge et al., 1996-a. Para pacientes com transtornos afetivos, o custo para o HD foi de 17.942 dólares, e de 28.508 dólares para os que foram submetidos ao regime de internação (Sledge et al., 1996-b).

Os dados desses dois trabalhos sugerem que embora a eficácia do tratamento em HD seja semelhante à da internação, o uso do HD pode baratear as despesas necessárias com o tratamento dos pacientes (Sledge et al., 1996-a e Sledge et al., 1996-b).

No primeiro estudo brasileiro a avaliar prospectivamente internações em HD (Lima e Botega, 2001), foram entrevistados 34 pacientes admitidos de 1/8/1997 a 31/7/1998 no HD da Faculdade de Medicina de Botucatu - Unesp. Os instrumentos utilizados para análise foram o Composite International Diagnostic Interview (CIDI), a Escala de Avaliação Psiquiátrica Breve (Brief Psychiatric Rating Scale-BPRS), a Escala de Avaliação da Incapacitação Psiquiátrica (Psychiatric Disability Assessment Schedule - DAS) e o Questionário para Obtenção de Dados Gerais. A média de idade de amostra foi de 37 anos, sendo $76 \%$ do sexo feminino e $24 \%$ do masculino; $44 \%$ dos pacientes haviam tido internações psiquiátricas prévias e a média de permanência no HD foi de 74 dias (Lima e Botega, 2001).

Foram atendidos pacientes com diagnósticos de transtornos afetivos (44,1\% da amostra), quadros psicóticos $(23,5 \%)$, transtornos ansiosos $(17,6 \%)$, quadros orgânicos $(11,8 \%)$ e dependências de álcool e drogas $(2,9 \%)$ (Lima e Botega, 2001). Houve melhora clínica em 79,4\% da amostra de 34 pacientes; $11,8 \%$ permaneceram inalterados e $8,8 \%$ pioraram. Ausência de internações psiquiátricas prévias e maior renda familiar foram fatores indicadores de melhor resposta, possivelmente por maior disponibilidade a psicofármacos mais caros, facilidades de acesso a serviços de saúde e melhores condições de vida (Lima e Botega, 2001).

\section{Hospital-dia (HD) para transtornos alimentares}

O primeiro trabalho a avaliar o HD para transtornos alimentares foi o conduzido por Danziger et al. (1988), que estudaram a evolução de 32 pacientes com anorexia nervosa, em Petah Tiqva (Israel), numa unidade pediátrica.

A equipe de atendimento era multidisciplinar, com atendimento psiquiátrico, pediátrico e recomendação de psicoterapia a todos os pacientes, bem como orientação aos pais, que acompanhavam os filhos durante o tratamento (Danziger et al., 1988).

O seguimento feito, em média nove meses após a alta, constatou que 27 ( $84 \%$ dos pacientes) mantinham o peso ideal, $2(6 \%)$ ficaram $4 \mathrm{~kg}$ acima do peso ideal, 2 $(6 \%)$ deles permaneciam em tratamento no hospital-dia e $1(3 \%)$ não ganhou peso adequado, sendo considerado uma falha terapêutica (Danziger et al., 1988).

Embora essa evolução não tenha sido comparada com outra modalidade de tratamento, os autores concluem que houve êxito no uso do HD e indicam-no antes de uma internação em enfermaria diuturna (Danziger et al, 1988).

$\mathrm{Na}$ América do Norte, as empresas de saúde particulares, responsáveis pelo tratamento de grande parcela da população local, dificultam internações psiquiátricas, em função do alto custo (Kaye et al., 1996). Há controle rígido da indicação à internação, do número de dias previstos de permanência no hospital, do tratamento e da evolução do paciente. Essas empresas encorajam alternativas para internação de transtornos psiquiátricos, como o HD. Para os transtornos alimentares, levam em conta a intensidade do quadro para determinar se o paciente fica internado ou recebe tratamento domiciliar ou faz HD ou tratamento ambulatorial. Esse é um problema para pacientes com transtornos alimentares, uma vez que um dos critérios universalmente aceitos para alta é o do índice de massa corpórea (IMC) mínimo de 19, o que pode levar à internação prolongada (Kaye et al., 1996).

O programa de HD em Toronto (Toronto General Hospital, The Day Hospital Group Treatment Program - DHP) surgiu da necessidade de tratar pacientes com transtornos alimentares, após o fechamento do hospital que os internava quando era necessário (Piran e Kaplan, 1990). O hospital geral de Toronto igualmente não dispunha de recursos para proporcionar esses leitos. O HD foi um modelo alternativo parar os transtornos alimentares (Piran et al., 1990).

O DHP iniciou suas atividades em 1985, sendo o pioneiro no atendimento a transtornos alimentares com um programa estruturado unicamente para tais patologias (Piran et al., 1989a; Piran et al., 1990). 
$\mathrm{O}$ HD funciona quatro dias por semana (de terça a sexta-feira), das 10 às 18 horas, sendo necessária a permanência dos pacientes durante todo o período; é possível o atendimento de até 12 pacientes (Piran e Kaplan, 1990). A permanência média é de 6 a 8 semanas para bulímicas e de 14 semanas para anoréxicas, sendo 1 mês o tempo mínimo de permanência e 4 meses o máximo. Os objetivos do HD são normalizar hábitos alimentares, aumentar o peso corporal quando esse estiver abaixo do esperado, corrigir distorções cognitivas provenientes dos transtornos alimentares e melhorar o funcionamento psicossocial dos pacientes (Kaye et al., 1996).

O programa, com exceção do atendimento psiquiátrico individual, desenvolve-se por meio de atendimento grupal, com aspectos comportamentais, cognitivos, psicodinâmicos e psicoeducacionais (Piran e Kaplan, 1990).

De 1985 a 1995, foram atendidos 587 pacientes, com 391 deles tendo terminado o programa, sendo $58,9 \%(n=230)$ com bulimia nervosa, $29,2 \%(n=122)$ com anorexia nervosa e $11,9 \%(n=46)$ com transtorno alimentar sem outra especificação (Kaye et al., 1996).

As pacientes com bulimia nervosa apresentavam à admissão, em média, 17 episódios de vômitos autoinduzidos e 10 episódios bulímicos por semana (Kaye et al., 1996). Já nas quatro últimas semanas de tratamento, houve diminuição de vômitos e episódios bulímicos para menos de uma vez por semana, mostrando a eficiência na redução dos sintomas a curto prazo (Kaye et al., 1996).

Após seguimento de dois anos, os ganhos obtidos no hospital-dia permaneciam, com $80 \%$ das pacientes tendo ficado boa parte desse período assintomática (Maddocks et al., 1992; Olmsted et al., 1994). Igualmente, não houve comparação com outra forma de tratamento (Maddocks et al., 1992; Olmsted et al., 1994).

As pacientes com anorexia nervosa apresentavamse no início do tratamento com índice de massa corporal de 16, em média, e, ao receber alta, haviam alcançado, em média, índice de 21 (Kaye et al., 1996).

Em um outro trabalho com os pacientes do HD de Toronto, observou-se melhora no funcionamento familiar, medido por meio da Family Assessment Measure (FAM), principalmente para grupos assintomáticos ao final do tratamento (Woodside et al., 1996). Esta melhora permaneceu após seguimento de dois anos da alta para esse grupo (Woodside et al., 1996).

Não houve identificação de fatores preditivos de resposta (Olmsted et al., 1996). Pacientes com melhora considerada rápida, lenta, parcial ou sem melhora não apresentavam diferenças significativas entre as variáveis estudadas (Olmsted et al., 1996).
Howard et al. (1999) estudaram retrospectivamente 59 pacientes do sexo feminino, transferidas de enfermaria psiquiátrica para HD, em Iowa City (Estados Unidos) sendo $30 \mathrm{com}$ anorexia nervosa subtipo restritivo, 19 com anorexia nervosa subtipo purgativo e $10 \mathrm{com}$ transtorno alimentar não-especificado. Quanto à avaliação do tratamento, a evolução no HD foi considerada boa, quando as pacientes tiveram alta ( $\mathrm{n}=45$, ou $76,3 \%$ da amostra), ou má, quando as pacientes do HD tiveram de ser novamente transferidas para internação $(\mathrm{n}=12$, ou $20,3 \%$ da amostra) ou se saíram de alta a pedido $(\mathrm{n}=$ 2 , ou $3,4 \%$ da amostra). As variáveis indicando risco para má evolução do quadro foram tempo de história maior do que seis anos, amenorréia há mais de dois anos e meio, $\mathrm{IMC} \leq 16,5$ à admissão e IMC $\leq 19$ na transferência da enfermaria para o HD (Howard et al., 1999).

O HD de Munique (Therapy Center for Eating Disorders - TCE, Max-Planck Institute) iniciou seu atendimento em 1989. O programa tem duração de quatro meses para até 24 pacientes. A equipe é composta por um psiquiatra, um clínico, três psicólogos, dois nutricionistas, três enfermeiros, um arteterapeuta e um terapeuta de dança. Funciona sete vezes por semana, das $8 \mathrm{~h}$ às $17 \mathrm{~h}$, sendo servidas duas refeições principais e dois lanches (Zipfel et al., 2002).

O tratamento é grupal, com atividades sobre aspectos nutricionais, imagem corporal, habilidades sociais, arteterapia e atendimento familiar (Zipfel et al., 2002). A clínica oferece a possibilidade de abrigar dez pacientes quando estes reportarem convívio familiar inadequado, não tiverem moradia ou apresentarem comorbidade com abuso ou dependência de drogas.

$\mathrm{O}$ atendimento de HD em Baton Rouge, nos Estados Unidos (Our Lady of the Lake Eating Disorders Program - OLOL), iniciou suas atividades em 1990 (Zipfel et al., 2002). Os critérios de admissão são: perda moderada de peso ( $5 \%$ a $15 \%$ abaixo do normal), rápida perda de peso (perda de $1 \mathrm{~kg}$ de peso por semana, nas últimas quatro semanas) e falha em tratamento ambulatorial (Zipfel et al., 2002).

A equipe é composta por um diretor médico psiquiatra, um psicólogo supervisor, três psicólogos com mestrado, dois nutricionistas, um terapeuta, um assistente social e um enfermeiro (Zipfel et al., 2002).

O funcionamento ocorre de segunda a sexta-feira, das $9 \mathrm{~h} 30$ às $17 \mathrm{~h} 30 \mathrm{em}$ dois dias da semana e das $9 \mathrm{~h} 30$ às $18 \mathrm{~h} 30$ nos outros três, com o objetivo de reduzir os custos, não sendo oferecido jantar nos dias em que o HD termina mais cedo (Zipfel et al., 2002). 
A média de duração do tratamento é de 50 dias e oferece capacidade para o atendimento de 5 a 13 pacientes simultaneamente (Zipfel et al., 2002). O local de atendimento é a enfermaria de internação, junto com os pacientes internados. O tratamento usa técnicas comportamentais e cognitivas com atividades em grupo para discussão de tópicos relacionados à doença e inclui um grupo de apoio para familiares e amigos dos pacientes (Zipfel et al., 2002).

As indicações para internação em enfermaria no OLOL são situações emergenciais clínicas ou psiquiátricas não especificadas, sendo as pacientes rapidamente transferidas para o $\mathrm{HD}$, mesmo que estejam abaixo do peso adequado (Zipfel et al., 2002). A readmissão para a enfermaria é feita se o ganho de peso, enquanto ela estiver freqüentando o $\mathrm{HD}$, for menor que $1 \mathrm{~kg}$ por semana (Zipfel et al., 2002).

Em um estudo realizado na Escócia, após 20 meses de seguimento, não foram encontradas diferenças nos resultados dos pacientes divididos aleatoriamente no HD ou na enfermaria; não há, no entanto, maior detalhamento do conteúdo programático desse HD (Zipfel et al., 2002).

No programa de transtornos alimentares do Hospital Johns Hopkins, nos Estados Unidos, a admissão ao HD segue-se à alta de enfermaria (Zipfel et al., 2002). O programa inicial é de sete vezes por semana, por 12 horas. Se há ganho de peso adequado, o paciente pode fazer uma das refeições fora. A freqüência ao programa diminui gradativamente à medida que $\mathrm{o}$ paciente obtém progressos, o período preparativo para a alta do HD é de três vezes por semana, 8 horas ao dia. Se o paciente não ganha peso, ele pode voltar ao início do tratamento (sete vezes por semana, 12 horas por dia), ou é novamente internado (Zipfel et al., 2002).

Em Sydney, faz-se um programa baseado em psicoterapia de grupo de apoio e interpessoal, quatro vezes por semana e durante 5 horas por dia. Pacientes tomavam café da manhã, almoço e chá da tarde. Os objetivos do tratamento não incluem mudança comportamental, normalização dos hábitos alimentares ou orientação nutricional. De 23 pacientes com anorexia nervosa que fizeram o programa, $95 \%$ deles perderam peso, num total de $2,1 \mathrm{~kg}$ de perda. Por causa disso, $64 \%$ foram readmitidos em enfermaria. $\mathrm{O}$ insucesso desse programa mostra a necessidade de enfatizar os tópicos de ganho de peso e mudança de atitudes em relação à alimentação (Zipfel et al., 2002).

\section{Hospital-dia do AMBULIM-IPqHCFMUSP}

O início do HD do Ambulatório de Bulimia e Transtornos Alimentares do Instituto de Psiquiatria do
Hospital das Clínicas da Faculdade de Medicina da Universidade de São Paulo (AMBULIM-IPqHCFMUSP) deu-se no final de 1999 no intuito de reduzir o número de internações e diminuir a recaída precoce após a internação.

O HD funciona na enfermaria feminina do Instituto de Psiquiatria - por razões econômicas e físicas -, não havendo outro espaço disponível para o atendimento. Há possibilidade de tratamento para até quatro pacientes, com duração de 12 semanas, de segunda a sexta-feira, das 10 às 18 horas.

São atendidos pacientes com diagnósticos de anorexia nervosa (subtipo purgativo ou restritivo), bulimia nervosa ou transtorno alimentar sem outra especificação, de acordo com o DSM-IV (APA, 1994).

A equipe multidisciplinar é composta por psiquiatras, psicólogos, nutricionista, arteterapeuta, enfermeiros e acompanhante terapêutico. Psiquiatras, nutricionista e equipe de enfermagem pertencem ao quadro de profissionais do Instituto de Psiquiatria do HCFMUSP e todos os outros participantes são voluntários.

As atividades são desenvolvidas individualmente nos atendimentos psiquiátrico e psicológico e em grupo nas demais atividades (arteterapia, grupo de atividade física, orientação vocacional, atendimento nutricional e grupo de leitura, entre outras). A equipe se reúne uma vez por semana para discussão dos casos em tratamento.

Desde o início do programa, e até a elaboração desse artigo, foram atendidos 24 pacientes no HDAMBULIM, cujos diagnósticos, de acordo com o DSMIV (1994), podem ser observados na tabela 1, e o número de admissões, na tabela 2. As atividades realizadas no HD do AMBULIM estão na tabela 3. Podemos observar dados dos programas de HD, citados acima, na tabela 4 e as atividades neles realizadas na tabela 5 .

Tabela 1 Diagnósticos dos pacientes do HD-AMBULIM (DSM-IV)

\begin{tabular}{lc}
\hline N & 24 \\
ANP & 5 \\
ANR & 3 \\
BN & 14 \\
TASOE & 2 \\
\hline $\mathrm{N}=$ número de pacientes \\
ANP = anorexia nervosa purgativa \\
ANR = anorexia nervosa restritiva \\
BN = bulimia nervosa \\
TASOE = transtornos alimentares sem especificação
\end{tabular}

Tabela 2 Número de admissões no HD-AMBULIM

\begin{tabular}{lc}
\hline Primeira admissão & 20 \\
Segunda admissão & 2 \\
\hline
\end{tabular}


Tabela 3 HD do AMBULIM - Grade horária

\begin{tabular}{|c|c|c|c|c|c|}
\hline \multicolumn{6}{|c|}{ Período: setembro a dezembro de 2002} \\
\hline HD & Segunda-feira & Terça-feira & Quarta-feira & Quinta-feira & Sexta-feira \\
\hline \multirow[t]{2}{*}{$7 \mathrm{~h} 45-8 \mathrm{~h} 30$} & & Reunião HD & & & \\
\hline & & Reunião geral & & & \\
\hline \multicolumn{6}{|l|}{$8 \mathrm{~h} 30-10 \mathrm{~h}$} \\
\hline $9 \mathrm{~h}-10 \mathrm{~h}$ & Psiquiatra & & Psiquiatra & & Psiquiatra \\
\hline $10 \mathrm{~h}-11 \mathrm{~h} 30$ & Atividade física & Terapia familiar & Projeto carmim & Eutonia & Oficina de cabeleireira \\
\hline $11 \mathrm{~h} 30-12 \mathrm{~h}$ & Almoço & Almoço & Almoço & Almoço & Almoço \\
\hline $12 \mathrm{~h}-13 \mathrm{~h}$ & Repouso & Repouso & Repouso & Repouso & Repouso \\
\hline $13 \mathrm{~h}-14 \mathrm{~h} 30$ & Nutrição & Terapia ocupacional & Grupo de leitura & & Oficina de origami \\
\hline $14 \mathrm{~h} 30-15 \mathrm{~h}$ & Lanche & Lanche & Lanche & Lanche & Lanche \\
\hline $15 \mathrm{~h}-16 \mathrm{~h} 30$ & $\begin{array}{c}\text { Aconselhamento } \\
\text { profissional }\end{array}$ & $\begin{array}{c}\text { Atendimento } \\
\text { psicoterapêutico }\end{array}$ & Grupo operativo & Artesanato & $\begin{array}{c}\text { Acompanhamento } \\
\text { terapêutico }\end{array}$ \\
\hline
\end{tabular}

Tabela 4 Programas de HD

\begin{tabular}{|c|c|c|c|c|c|c|}
\hline & Ano de início & Dias/S. & Horário & $\mathrm{N}$ & $\mathrm{T}$ & Adm. \\
\hline DHP-Toronto & 1985 & 4 & $10 \mathrm{~h}-18 \mathrm{~h} 15$ & 12 & 2 a 4 meses & $\begin{array}{l}\text { T.A., F.T.A, apoio familiar - } \\
\text { concordar com tratamento grupal }\end{array}$ \\
\hline TCE-Munique & 1989 & 7 & $8 \mathrm{~h}-17 \mathrm{~h}$ & 24 & 4 meses & T.A., 10 vagas $\mathrm{p} /$ dormir \\
\hline OLOL-Baton Rouge & 1990 & 5 & $\begin{array}{c}9 \mathrm{~h} 30-17 \mathrm{~h} 30 \text { ou } \\
18 \mathrm{~h} 30\end{array}$ & 5 a 13 & 50 dias & $\begin{array}{c}\text { T.A., F.T.A. } \\
\text { Peso } 5 \% \text { a } 10 \% \text { abaixo do normal } \\
\text { Perda de } 1 \mathrm{~kg} / \text { semana por } \\
4 \text { semanas seguidas }\end{array}$ \\
\hline AMBULIM-Ipq & 1999 & 5 & $10 \mathrm{~h}-18 \mathrm{~h}$ & 4 & 12 semanas & $\begin{array}{c}\text { T.A., F.T.A., transição da } \\
\text { enfermaria para ambulatório }\end{array}$ \\
\hline Johns Hopkins & ND & 7 & $7 h-19 h$ & ND & ND & $\begin{array}{l}\text { T.A., transição da enfermaria } \\
\text { para ambulatório }\end{array}$ \\
\hline
\end{tabular}

Adm. = admissão ao tratamento

Dias $/$ S. = dias de funcionamento por semana

F.T.A. $=$ falha de tratamento ambulatorial

$\mathrm{N}=$ número de pacientes atendidos

$\mathrm{T}=$ tempo de permanência no $\mathrm{HD}$

T.A. $=$ Transtornos alimentares

$\mathrm{ND}=$ dado não disponível

Tabela 5 Atividades dos HDs

\begin{tabular}{ll}
\hline AMBULIM & $\begin{array}{l}\text { Nutrição; atividade física; terapia familiar; eutonia; acompanhante terapêutico; terapia ocupacional; oficina de } \\
\text { cabeleireira; projeto carmim (oficina de artes plásticas); grupo de leitura; oficina de origami; artesanato; } \\
\text { aconselhamento profissional; psicoterapia individual; grupo operativo. }\end{array}$ \\
\hline DHP & $\begin{array}{l}\text { Discussão sobre refeições; nutrição; revisão da semana que passou; culinária; grupo educacional; encontros } \\
\text { comunitários; planejamento do final de semana; atividade física; imagem corporal. }\end{array}$ \\
\hline OLOL & $\begin{array}{l}\text { Discussão sobre refeições; nutrição; grupo processual; grupo de atividades; grupo educacional familiar; } \\
\text { planejamento de refeições; assertividade; grupo de desenvolvimento de habilidades sociais; grupo de terapia } \\
\text { comportamental-cognitiva; imagem corporal. }\end{array}$ \\
\hline TCE & $\begin{array}{l}\text { Discussão sobre refeições; grupo de aconselhamento; arteterapia; grupo educacional familiar; grupo de } \\
\text { desenvolvimento de habilidades sociais; grupo de relaxamento; imagem corporal. }\end{array}$ \\
\hline
\end{tabular}




\section{Discussão}

Desde seu aparecimento na antiga União Soviética por razões econômicas e provavelmente ideológicas, houve a adoção do modelo de HD em muitas partes do mundo como parte da estrutura de atendimento hospitalar, integrado à enfermaria psiquiátrica ou atuando isoladamente.

Um dos motivos de seu surgimento foi ser uma alternativa à internação, com vários programas psicoterápicos e de reabilitação, diferente da estrutura hospitalar tradicional (Dick et al., 1985[b]).

Pacientes alocados aleatoriamente em HD ou em enfermaria tiveram tratamentos igualmente eficazes (Herz et al., 1971 ; Hirsch et al., 1979; Horvitz-Lennon et al., 2001).Tanto os pacientes como seus familiares preferiram o tratamento no HD em vez da internação tradicional (Dick et al., 1985[a]; Horvitz-Lennon et al., 2001).

O tratamento em HD mantém a autonomia do paciente e tenta promover a utilização das técnicas desenvolvidas para controle dos sintomas da doença, ao retorno do paciente para seu ambiente domiciliar. Nesse ambiente estão os desencadeantes que predispõem a alterações no padrão alimentar e no controle do peso e da forma corporal. O contato diário com a equipe do HD pode oferecer detecção precoce e possibilidade de intervenção mais rápida.

Os pacientes não sentem que o tratamento é punitivo e há redução na dependência, na hostilidade e nas atitudes inadequadas para com funcionários quando se compara com o que ocorre durante internação, uma vez que não precisam dormir lá ou ter que permanecer nos fins de semana (Dibella et al., 1982).

Como os pacientes retornam para suas casas à noite e nos fins de semana, eles mantêm intacto o relacionamento com sua família e amigos, não ficando limitados à vida intra-hospitalar somente (Dibella et al., 1982).

Muitos programas utilizam-se de atendimentos grupais por razões econômicas, propiciando apoio mútuo, além de diminuir a sensação de vergonha e impotência diante da doença.

Há evidentes razões econômicas e vantagens financeiras para tratarmos transtornos alimentares em $\mathrm{HD}$, uma vez que não há necessidade de se manter equipe de atendimento por 24 horas, além de possibilitar economia das despesas hospitalares de manutenção em geral (Kaye et al., 1996).

Kaye et al. (1996) mostram que o custo por paciente no HD de Toronto representa cerca de $25 \%$ a $30 \%$ do custo de uma internação em enfermaria. Para moradores da região da cidade, é de 160 dólares por dia; a internação na enfermaria eleva o valor para 507 dólares.

Em relação ao OLOL-Baton Rouge, houve melhora clínica para pacientes do HD e da enfermaria, sem diferenças significativas entre ambos, com custo de tratamento no HD de $48 \%$ do valor gasto na enfermaria (Zipfel et al., 2002).

Alguns pacientes têm quadros mais graves que exigem uma permanência de 24 horas por dia, com estrutura de internação, portanto. Se existe presença de comportamentos auto-agressivos, a internação pode ser necessária.

Pode haver dificuldade em realizar um tratamento que usa técnicas grupais para quem tem dificuldade em relacionar-se com outras pessoas (Piran e Kaplan, 1990).

As indicações para internação no HD do AMBULIM são falha no tratamento ambulatorial, transição da internação tradicional para retorno ao lar e concordância em realizar atividades grupais. As contra-indicações para admissão são: ideação suicida, desnutrição importante que impossibilita acompanhamento externo e abuso de álcool e drogas, o qual interfira na melhora do padrão alimentar.

A equipe de atendimento precisa ser exaustivamente treinada e adquirir habilidade diante da vigilância que deve ser feita (em especial, durante e imediatamente após as refeições) e da participação nas formas de atendimento. Para Kaye et al. (1996) isso é desvantajoso, pois significa maior gasto e tempo de treinamento diante da equipe psiquiátrica habitual; porém a questão da internação mais rápida e vigiada versus uma abordagem mais leniente para pacientes com comportamentos purgativos ainda está em aberto e a maioria dos modelos de internação prefere um esquema mais draconiano.

As metas do tratamento são: a normalização do padrão alimentar com a interrupção dos episódios bulímicos e práticas compensatórias (medidas purgativas e restritivas); orientação nutricional com ganho de peso, se necessário, e identificação de alterações psicológicas que reforcem o transtorno alimentar.

São realizados tratamentos biológicos, que incluem psicofármacos, estabilização da parte clínica e nutricional (Becker et al., 1999). A oferta calórica é progressivamente aumentada até atingir o nível adequado para a altura e idade do paciente (Piran et al., 1989[a]).

As intervenções sociais incluem aconselhamento vocacional, discussão sobre moradia e locais de suporte na comunidade. 
Psicoterapia é de extrema importância para a recuperação dos pacientes (Kaye et al., 2000). As modalidades que mais contribuem são de enfoque psicoeducacional (Connors et al., 1984; Long e Cordle, 1982), comportamental-cognitivo (Wilson et al.,1997) e terapia interpessoal (Fairburn et al., 1991). O apoio psicológico inclui terapia familiar ou de casal, englobando parentes que tenham afinidade com o paciente. Podem-se realizar vários tipos de atividades grupais ao longo da semana, oferecendo estruturação do tempo livre e alternativas às distorções causadas pelos transtornos alimentares, $\mathrm{o}$ que possibilita maior continência ao usuário.

Os pacientes e a família devem estar motivados a se envolver num tratamento mais intensivo e sua com participação ativa.

Em relação aos modelos de atendimento de HD em alguns centros de tratamento de transtornos alimentares, observamos o mesmo enfoque na abordagem multidisciplinar e no predomínio de atividades em grupo. O AMBULIM e o OLOL-Baton Rouge são os únicos que oferecem psicoterapia individual, o que para nossos pacientes é muito importante, pois geralmente há longa espera para consegui-la gratuitamente em serviços específicos.

Há diferenças no número de dias de atendimento, com serviços funcionando quatro vezes por semana (no DHP-Toronto e em Sydney), cinco vezes (AMBULIM e OLOL) e sete vezes (Johns Hopkins e TCE-Munique).

Surpreendentemente, porém, a redução dos dias de atendimento de cinco para quatro vezes por semana feita pelo DHP de Toronto não provocou mudança no ritmo de ganho de peso para as pacientes com anorexia nervosa. Entre os pacientes com bulimia nervosa, que tinham mais do que oito episódios bulímicos e de vômitos auto-induzidos à admissão, as taxas de remissão eram menores no grupo que freqüentou o HD por quatro dias. Dados psicométricos que avaliam atitudes alimentares e auto-estima não indicaram diferenças nos dois grupos (Zipfel et al., 2002).

O TCE possui maior quantidade de vagas para tratamento, com 24 pacientes podendo ser atendidas simultaneamente, enquanto o DHP tem 12 vagas e o AMBULIM tem 4.

A estrutura de atendimento é diferente nos diversos HDs. No AMBULIM, no OLOL e no Johns Hopkins, o HD é um passo intermediário entre a internação e o tratamento ambulatorial, enquanto no DHP e no TCE ele é uma alternativa à internação.

A possibilidade de alojar pacientes à noite é uma característica somente encontrada no TCE, facilitando o tratamento de pacientes que moram muito longe ou não têm apoio familiar, resguardando os que apresentam problemas com drogas.
Estudos realizados no DHP indicaram bons resultados no tratamento de transtornos alimentares em HD. Há ganho de peso em pacientes com anorexia nervosa, diminuição da freqüência de episódios bulímicos e de vômitos auto-induzidos para pacientes com bulimia nervosa a curto e médio prazo (Maddocks et al., 1992; Olmsted et al., 1994).

\section{Considerações finais}

O objetivo deste estudo foi revisar a literatura a respeito de tratamento de transtornos psiquiátricos em $\mathrm{HD}$, com particular interesse nos transtornos alimentares, e apresentar o serviço desenvolvido no Ambulatório de Bulimia e Transtornos Alimentares do Instituto de Psiquiatria do Hospital das Clínicas da Faculdade de Medicina da Universidade de São Paulo (AMBULIMIPqHCFMUSP).

É uma modalidade de atendimento com bons resultados para o paciente com anorexia nervosa ou bulimia nervosa, desde que respeitadas as indicações e as contra-indicações para sua admissão. É importante identificarmos fatores que apontem quem não se beneficiaria com o HD. O estudo para tal deveria ser prospectivo e comparando pacientes do HD com pacientes internados em enfermaria, com escolha aleatória. Alguns pacientes, no entanto, claramente precisam ser internados em enfermaria, estando sob maiores cuidados, o que dificulta a obtenção da amostra; além do mais, a oferta de leitos de internação é pequena.

Dependendo da gravidade do quadro clínico dos pacientes, tem-se a impressão de que os esforços da equipe são inúteis, podendo levar à exaustão e à desmobilização. Para evitar o desgaste, é importante haver coesão, além de supervisões constantes, a fim de se discutirem as dificuldades e como superá-las (Piran e Kaplan, 1990). A experiência do HD-AMBULIM tem nos mostrado que as reuniões semanais da equipe e o contato entre os membros do grupo nos outros períodos são suficientes para evitar as dificuldades citadas acima.

Esperamos continuar com nosso trabalho e, no futuro, estudar questões ainda não bem resolvidas quanto ao atendimento de transtornos alimentares em hospital-dia, como avaliação a longo prazo dos resultados clínicos do HD e o custo para mantê-lo, além de comparar a eficácia desse tipo de atendimento com o tratamento ambulatorial, principalmente para pacientes com bulimia nervosa, e com internação em enfermaria, para as pacientes com anorexia nervosa. A quantidade ideal de horas a ser oferecida durante o tratamento no HD ainda não está plenamente determinada, sendo necessários mais estudos nesse sentido. 


\section{Referências bibliográficas}

Americam Psychiatric Association (APA). - Diagnostic and Statistical Manual Of Mental Disorders. 4th ed. Washington, DC, Author, 1994.

ARIE, T. - Day care in geriatric psychiatry. Gerontologia Clinica 17: 319, 1975.

Becker, A.E.; Grinspoon, S.K.; Klibanski, A.; Herzog, D.B. - Eating Disorders. New England Journal of Medicine 340: 1092-1098, 1999.

CAMERON, D.E. - The Day-hospital: Experimental Forms of Hospitalization for Patients. Modern Hospital 69: 60-2, 1947.

CASARINo, J.P.; Wilner, M.; MAXey, J.T. - American Association for Partial Hospitalization (AAPH): Standards and Guidelines for Partial Hospitalization. International Journal of Partial Hospitalization 1: 5-21, 1982.

Connors, M.E.; Johnson,C.L.; Stuckey, M.D. - Treatment of Bulimia With Brief Psychoeducational Group Therapy. American Journal of Psychiatry 141: 1512-6, 1984.

CRAFT, M. - Psychiatric Day Hospitals. American Journal of Psychiatry 116: 251-4, 1959

Danziger, Y.; Carcl, C.A.; Varsano, I.; Tyano, S.; Minouni, M. Parental Involvement In Treatment Of Patients With Anorexia Nervosa in a pediatric Day-Care Unit. Pediatrics 81: 159-62, 1988.

Dibella, G.A.W.; Weitz, G.W.; Poynter-Berg, D.; Yurmark, J.L. Handbook of Partial Hospitalization. New York: Brunner-Mazel, 1982.

Dick, P.; CAmeron, L.; Cohen, D.; Barlow, N.; Ince, A. - Day and Fulltime Psychiatric Treatment: a Controlled Comparison. British Journal of Psychiatry 147: 246-50, 1985(a)

Dick, P.; Ince, A; BARLow, N. - Day Treatment: Suitability and Referral Procedures. British Journal of Psychiatry 147: 250-3, 1985(b).

Fairburn, C.G.; Jones, R.; Peveler, R.C.; Carr, S.J.; Solomon, R.A.; Oconnor, M.E.; Burton, J.; Hope, R.A. - Three Psychological Treatments for Bulimia Nervosa: A Comparative Trial. Archives of General Psychiatry 48: 463-9, 1991.

Fox, V.; Lowe,G.D. - Day Hospital Treatment of the Alcoholic Patient. Quarterly Journal of the Studies of Alcohol 29: 634-41, 1968.

Gudeman, J.E.; Shore, M.F.; Dicjey, B. - Day Hospitalization and an inn Instead of Inpatient Care for Psychiatric Patients. The New England Journal of Medicine 308: 749-53, 1983.

Herz, M.I.; Endicott, J.; Spitzer, R.I.; Mesnikoff, A. - Day Versus inpatient Hospitalization, a Controlled study. American Journal of Psychiatry 127: 1371-82, 1971.

Hirsch, S.R.; Platt, S.; Knights, A.; Wayman, A. - Shortening Hospital Stay For Psychiatric Care: Effects on Patients and Their Families. British Medical Journal I: 442-6, 1979.

Horvitz-lennon, M.; Normand, S.L.T.; Gaccione, P.; Frank, R.G. Partial versus Full Hospitalization for Adults in Psychiatric Distress: a Systematic Review of the Published Literature (1957-1997). American Journal of Psychiatry 158: 676-85, 2001.

Howard, W.T.; Evans, K.K.; Quintero-howard, C.V.; Bowers, W.A.; Andersen, A.E. - Predictors of Success or Failure of Transition to day Hospital Treatment for Inpatients with Anorexia Nervosa. American Journal of Psychiatry 156: 1697-702, 1999.
Kaye, W.H.; Kaplan, A.S.; Zucker, M.L. - Treating eating-disorder Patients in a Managed Care Environment. The Psychiatric Clinics of North America 19: 793-810, 1996.

Kaye, W.H.; Klump, K.L.; Frank, G.K.W.; STROBer, M. - Anorexia and Bulimia Nervosa. Annual Reviews of Medicine 51:299-313, 2000.

Lima, M.C.P.; Botega, N.J. - Hospital-dia: para quem e para quê? Revista Brasileira de Psiquiatria 23: 195-9, 2001.

Long, G.C.; Cordle, C.J. - Psychological Treatment of Binge-eating and Self-induced Vomiting. Journal of Medical Psychology 55: 139-45, 1982.

Maddocks, S.E.; Kaplan, A.S.; Wodside, D.B.; Langdon, L.; PiRan, N. - Two Year Follow-up of Bulimia Nervosa: the Importance of Abstinence as the Criterion of Outcome. International Journal Eating Disorders 12: 133-41, 1992.

Neisworth, J.T.; Feeg,V.D. - Partial Hospitalization for Mentally Retarded Citizens. In: Luber, R.F. (ed.): Partial Hospitalization: a Current Perspective. Plenum Press, New York, pp. 46, 1979.

Olmsted, M.P., Kaplan, A.S.; Rockert, W. - Rate and prediction of Relapse in Bulimia Nervosa. American Journal of Psychiatry 151: 728-43, 1994.

Olmsted, M.P.; Kaplan, A.S.; Rockert, W. \& Jacobsen, M. - Rapid Responders to Intensive Treatment of Bulimia Nervosa. International Journal of Eating Disorders 19: 279-85, 1996.

Piran, N.; Kaplan, A.; Kerr, A.; Shekter-Wolfson, L.; Winocur, J.; Gold, E. \& GarfinKel, P.E. - A Day Hospital Program for Anorexia Nervosa and Bulimia . International Journal of Eating Disorders 8: 511-21, 1989(a).

Piran, N.; Langdon, L.; Kaplan, A.; Garfinkel, P.E. - Evaluation of a Day Hospital Program for Eating Disorders. International Journal of Eating Disorders 8: 523-32, 1989(b).

Piran, Niva; Kaplan, Allan S. - A Day Hospital Group Treatment Program for Anorexia Nervosa and Bulimia Nervosa. Brunner/ Mazel, New York, 1990.

Sledge, W.H.; Tebes, J.; Rakfeldt, J.; Davidson, L.; Lyons, L.; Druss, B. - Day Hospital/Crisis Respite Care versus Inpatient Care, part I: clinical outcomes. American Journal of Psychiatry 153: 106573, 1996(a).

Sledge, W.H.; Tebes, J.; WolfF, N.; Helminiak, T.w. - Day Hospital/ Crisis Respite Care versus Inpatient Care, part ii: service utilization and costs. American Journal of Psychiatry 153: 1074-83, 1996(b).

Wilson, G.T.; Fairburn, C.G.; Agras, W.S. - Cognitive Behavioural treatment for bulimia nervosa. In: GARNER, D.M. \& GARFINKEL, P.E. (eds.): Handbook of Treatment for Eating Disorders. Guilford Press, New York, pp. 94-144, 1997.

Woodside, D. B.; Lackstrom, J.; Shekter-wolfson, L.; HeinnaA M. Long-tern Follow-up of Patient-reported Family Functioning in Eating Disorders After Intensive Day-hospital Treatment. Journal of Psychosomatic Research 4: 269-77, 1996.

Zipfel, S.; Reas, D.L.; Thorton, C.; Olmsted, M.P.; Williamson, D.A.; Gerlinghoff, M.; Herzog, W.; Beaumont, P.J. - Day Hospitalization Programs for Eating Disorders: a Systematic Review of the Literature. International Journal of Eating Disorders 31: 105-17, 2002. 